\title{
Characterizations of Multiparameter Besov and Triebel-Lizorkin Spaces Associated with Flag Singular Integrals
}

\author{
Xinfeng Wu and Zongguang Liu \\ Department of Mathematics, China University of Mining and Technology (Beijing), \\ Beijing 100083, China \\ Correspondence should be addressed to Xinfeng Wu, wuxf@cumtb.edu.cn
}

Received 25 February 2012; Accepted 9 April 2012

Academic Editor: Yongsheng S. Han

Copyright (C) 2012 X. Wu and Z. Liu. This is an open access article distributed under the Creative Commons Attribution License, which permits unrestricted use, distribution, and reproduction in any medium, provided the original work is properly cited.

We introduce the inhomogeneous multiparameter Besov and Triebel-Lizorkin spaces associated with flag singular integrals via the Littlewood-Paley-Stein theory. We establish difference characterizations and Peetre's maximal function characterizations of these spaces.

\section{Introduction and Main Results}

The flag singular integral operators were first introduced by Müller, Ricci, and Stein when they studied the Marcinkiewicz multiplier on the Heisenberg groups in [1]. To study the $\square_{b^{-}}$ complex on certain CR submanifolds of $\mathbb{C}^{n}$, in 2001, Nagel et al. [2] studied a class of product singular integrals with flag kernel. They proved, among other things, the $L^{p}$ boundedness of flag singular integrals. More recently, Nagel et al. in $[3,4]$ have generalized these results to a more general setting, namely, homogeneous group. For other related results, see $[5,6]$.

For $0<p \leq 1$, Han and Lu [7] developed Hardy spaces $H_{\mathfrak{F}}^{p}\left(\mathbb{R}^{n} \times \mathbb{R}^{m}\right)$ with respect to the flag multiparameter structure via the discrete Littlewood-Paley-Stein analysis and discrete Calderón's identity and proved the $H_{\mathfrak{F}}^{p}\left(\mathbb{R}^{n} \times \mathbb{R}^{m}\right) \rightarrow H_{\mathfrak{F}}^{p}\left(\mathbb{R}^{n} \times \mathbb{R}^{m}\right)$ and $H_{\mathfrak{F}}^{p}\left(\mathbb{R}^{n} \times \mathbb{R}^{m}\right) \rightarrow L^{p}\left(\mathbb{R}^{n} \times\right.$ $\left.\mathbb{R}^{m}\right)$ boundedness for flag singular integral operators. The duality of $H_{\mathfrak{F}}^{p}\left(\mathbb{R}^{n} \times \mathbb{R}^{m}\right)$ was also established. More recently, Ding et al. studied the homogeneous Besov spaces and TriebelLizorkin spaces associated with flag singular integrals in [8] and proved the boundedness of flag singular integrals on these spaces. Similar results can also be found in [9].

The aim of this paper is to give the new difference characterization as well as Petree's maximal function characterization of multiparameter Besov and Triebel-Lizorkin spaces associated with flag singular integrals, which reflect that the Besov spaces and TriebelLizorkin spaces have flag multiparameter structure. These characterizations are established for 
the inhomogeneous Besov and Triebel-Lizorkin spaces, but the argument goes through with only minor alterations in the homogeneous ones introduced in [8].

In order to describe more precisely questions and results studied in this paper, we begin with basic notations and notions. Let $\psi^{(1)} \in \mathcal{S}\left(\mathbb{R}^{n} \times \mathbb{R}^{m}\right)$ with

$$
\begin{gathered}
\operatorname{supp} \widehat{\psi^{(1)}} \subset\left\{(\xi, \eta) \in \mathbb{R}^{n} \times \mathbb{R}^{m}: \frac{1}{2} \leq|\xi, \eta| \leq 2\right\}, \\
\sum_{j \in \mathbb{Z}}\left|\widehat{\psi^{(1)}}\left(2^{-j} \xi, 2^{-j} \eta\right)\right|^{2}=1 \quad \forall(\xi, \eta) \in \mathbb{R}^{n} \times \mathbb{R}^{m} \backslash\{(0,0)\},
\end{gathered}
$$

and let $\psi^{(2)} \in \mathcal{S}\left(\mathbb{R}^{m}\right)$ with

$$
\begin{aligned}
& \text { supp } \widehat{\psi^{(2)}} \subset\left\{\eta \in \mathbb{R}^{m}: \frac{1}{2} \leq|\eta| \leq 2\right\} \\
& \sum_{k \in \mathbb{Z}}\left|\widehat{\psi^{(2)}}\left(2^{-k} \eta\right)\right|^{2}=1 \quad \forall \eta \in \mathbb{R}^{m} \backslash\{0\} .
\end{aligned}
$$

Let $\psi_{j}^{(1)}(x, y)=2^{j n} \psi^{(1)}\left(2^{j} x, 2^{j} y\right), \psi_{k}^{(2)}(y)=2^{k m} \psi^{(2)}\left(2^{k} y\right)$, and

$$
\psi_{j, k}(x, y)=\psi_{j}^{(1)} *_{2} \psi_{k}^{(2)}(x, y)=\int \psi_{j}^{(1)}(x, y-z) \psi_{k}^{(2)}(z) d z
$$

then, for $f \in L^{2}\left(\mathbb{R}^{n} \times \mathbb{R}^{m}\right)$, the following Calderón's reproducing formula holds:

$$
f=\sum_{j \in \mathbb{Z}} \sum_{k \in \mathbb{Z}} \psi_{j, k} * \psi_{j, k} * f
$$

where the series converges in $L^{2}\left(\mathbb{R}^{n} \times \mathbb{R}^{m}\right)$.

A Schwartz function $f \in \mathcal{S}\left(\mathbb{R}^{n+2 m}\right)$ is said to be a product test function in $\mathcal{S}_{\infty}\left(\mathbb{R}^{n+m} \times \mathbb{R}^{m}\right)$ if it satisfies

$$
\int_{\mathbb{R}^{m} \times \mathbb{R}^{n}} f(x, y, z) x^{\alpha} y^{\beta} d x d y=\int_{\mathbb{R}^{m}} f(x, y, z) z^{\gamma} d z=0
$$

for all multi-indices $\alpha \in \mathbb{N}^{n}, \beta, \gamma \in \mathbb{N}^{m}$.

Definition 1.1. A function $f(x, y)$ defined on $\mathbb{R}^{n} \times \mathbb{R}^{m}$ is said to be a test function in $\mathcal{S}_{\mathfrak{F}}\left(\mathbb{R}^{n} \times \mathbb{R}^{m}\right)$ if there exists a function $f^{\#} \in \mathcal{S}_{\infty}\left(\mathbb{R}^{m+n} \times \mathbb{R}^{m}\right)$ such that

$$
f(x, y)=\int_{\mathbb{R}^{m}} f^{\#}(x, y-z, z) d z,
$$

and the seminorm of $f$ is defined by

$$
\|f\|_{\mathfrak{F}, \alpha, \beta}=\inf \left\{\left\|f^{\#}\right\|_{\alpha, \beta}: \text { for all representations of } f \text { in (1.7) }\right\} \text {, }
$$


where $\|\cdot\|_{\alpha, \beta}$ denote the seminorm in $\mathcal{S}\left(\mathbb{R}^{n+2 m}\right)$. Denote by $\mathcal{S}_{\mathfrak{F}}\left(\mathbb{R}^{n} \times \mathbb{R}^{m}\right)$ the dual of $\mathcal{S}_{\mathfrak{F}}\left(\mathbb{R}^{n} \times\right.$ $\left.\mathbb{R}^{m}\right)$.

Choose a Schwartz function $\varphi$ on $\mathbb{R}^{n} \times \mathbb{R}^{m}$ such that

$$
|\widehat{\varphi}(\xi, \eta)|^{2}=1-\sum_{j=1}^{\infty} \sum_{k=1}^{\infty}\left|\widehat{\psi^{(1)}}\left(2^{-j} \xi_{1}, 2^{-j} \xi_{2}\right)\right|^{2}\left|\widehat{\psi^{(2)}}\left(2^{-k} \xi_{2}\right)\right|^{2}
$$

Note that $\varphi \in \mathcal{S}_{\mathfrak{F}}\left(\mathbb{R}^{n} \times \mathbb{R}^{m}\right)$ with Fourier transform is supported in $\{(\xi, \eta):|(\xi, \eta)| \leq 1\}$. Define the operator $S_{0}$ by $S_{0} f=\varphi * f, f \in \mathcal{S}_{\mathfrak{F}}^{\prime}\left(\mathbb{R}^{n} \times \mathbb{R}^{m}\right)$.

For $f \in L^{2}\left(\mathbb{R}^{n} \times \mathbb{R}^{m}\right)$, by taking the Fourier transform,

$$
f=\varphi * \varphi * f+\sum_{j=1}^{\infty} \sum_{k=1}^{\infty} \psi_{j, k} * \psi_{j, k} * f
$$

where the series converges in $L^{2}\left(\mathbb{R}^{n} \times \mathbb{R}^{m}\right)$ norm.

Now, we introduce the definition of inhomogeneous Besov spaces and TriebelLizorkin spaces associated with flag singular integrals.

Definition 1.2. Let $\alpha, \beta \in(-\infty, \infty)$ and $p, q \in(1, \infty)$. The inhomogeneous Triebel-Lizorkin space associated with flag singular integrals $\mathcal{F}_{p, q}^{\alpha, \beta}\left(\mathbb{R}^{n} \times \mathbb{R}^{m}\right)$ is defined to be the collection of all $f \in$ $\mathcal{S}_{\mathfrak{F}}\left(\mathbb{R}^{n} \times \mathbb{R}^{m}\right)$ such that

$$
\|f\|_{\mathcal{F}_{p, q}^{\alpha, \beta}\left(\mathbb{R}^{n} \times \mathbb{R}^{m}\right)}^{\psi}:=\left\|S_{0} f\right\|_{L^{p}\left(\mathbb{R}^{n} \times \mathbb{R}^{m}\right)}+\left\|\left(\sum_{j=1}^{\infty} \sum_{k=1}^{\infty} 2^{j \alpha q} 2^{k \beta q}\left|\psi_{j, k} * f\right|^{q}\right)^{1 / q}\right\|_{L^{p}\left(\mathbb{R}^{n} \times \mathbb{R}^{m}\right)}<\infty
$$

and the inhomogeneous Besov space associated with flag singular integrals $\boldsymbol{B}_{p, q}^{\alpha, \beta}\left(\mathbb{R}^{n} \times \mathbb{R}^{m}\right)$ is defined to be the collection of all $f \in \mathcal{S}_{\mathfrak{F}}{ }^{\prime}\left(\mathbb{R}^{n} \times \mathbb{R}^{m}\right)$ such that

$$
\|f\|_{\mathbb{B}_{p, q}^{\alpha, \beta}\left(\mathbb{R}^{n} \times \mathbb{R}^{m}\right)}^{\psi}:=\left\|S_{0} f\right\|_{L^{p}\left(\mathbb{R}^{n} \times \mathbb{R}^{m}\right)}+\left(\sum_{j=1}^{\infty} \sum_{k=1}^{\infty} 2^{j \alpha q} 2^{k \beta q}\left\|\psi_{j, k} * f\right\|_{L^{p}\left(\mathbb{R}^{n} \times \mathbb{R}^{m}\right)}^{q}\right)^{1 / q}<\infty
$$

Throughout this paper, we always work on $\mathbb{R}^{n} \times \mathbb{R}^{m}$ for some fixed $n, m$ and use $\mathcal{F}_{p, q}^{\alpha, \beta}$ to denote $\mathcal{F}_{p, q}^{\alpha, \beta}\left(\mathbb{R}^{n} \times \mathbb{R}^{m}\right)$, similarly for $\mathcal{S}_{\mathfrak{F}}, \mathcal{B}_{p, q}^{\alpha, \beta}$, and so forth. We would like to point out that the multiparameter structures are involved in the definitions of $\boldsymbol{B}_{p, q}^{\alpha, \beta}$ and $\mp_{p, q}^{\alpha, \beta}$. The following result shows that the definition of the Besov spaces $\mathcal{B}_{p, q}^{\alpha, \beta}$ and Triebel-Lizorkin spaces $\mathcal{F}_{p, q}^{\alpha, \beta}$ is independent of the choice of $\left(\psi^{(1)}, \psi^{(2)}, \varphi\right)$; thus, the Besov spaces $\mathbb{B}_{p, q}^{\alpha, \beta}$ and the Triebel-Lizorkin spaces $\mathcal{F}_{p, q}^{\alpha, \beta}$ are well defined. 
Theorem 1.3. If $\theta_{j, k}$ satisfies the same conditions as $\psi_{j, k}$, and $\phi$ is defined similar to (1.8) with $\psi$ replaced by $\theta$, then for $\alpha, \beta \in \mathbb{R}$ and $p, q \in(1, \infty)$ and $f \in \mathcal{S}_{\mathfrak{F}}$,

$$
\|f\|_{\mathcal{F}_{p, q}^{\alpha, \beta}}^{\theta} \sim\|f\|_{\mathcal{F}_{p, q}^{\alpha, \beta \prime}}^{\psi} \quad\|f\|_{\mathcal{B}_{p, q}^{\alpha, \beta}}^{\theta} \sim\|f\|_{\mathcal{B}_{p, q}^{\alpha, \beta}}^{\psi}
$$

Remark 1.4. As the classical case, it is not hard to show that $\|\cdot\|_{B_{p, q}^{\alpha, \beta}}^{\psi}$ and $\|\cdot\|_{\mathcal{F}_{p, q}^{\alpha, \beta}}^{\psi}$ are norms of $\mathcal{B}_{p, q}^{\alpha, \beta}$ and $\mathcal{F}_{p, q}^{\alpha, \beta}$, respectively. Moreover, $\mathcal{B}_{p, q}^{\alpha, \beta}$ and $\mathcal{F}_{p, q}^{\alpha, \beta}$ are complete with respect to these norms and hence are Banach spaces. We omit the details.

Throughout this paper, we use the notations $j \wedge k=\min \{j, k\}$ and $j \vee k=\max \{j, k\}$. We introduce the following flag multi-parameter Peetre maximal functions (with respect to $\psi$ ). For $j, k \in \mathbb{Z}_{+}$and $\vec{b}=\left(b_{1}, b_{2}\right) \in \mathbb{R}_{+} \times \mathbb{R}_{+}$, define

$$
\psi_{\vec{b}, j, k}^{*}(f)(x, y)=\sup _{(u, v) \in \mathbb{R}^{n} \times \mathbb{R}^{m}} \frac{\left|\psi_{j, k} * f(x-u, y-v)\right|}{\left(1+2^{j}|u|\right)^{b_{1}}\left(1+2^{j \wedge k}|v|\right)^{b_{2}}} .
$$

For $j=k=0$, define

$$
\varphi_{\vec{b}, 0,0}^{*}(f)(x)=\sup _{y \in \mathbb{R}^{n}} \frac{|\varphi * f(x-u, y-v)|}{(1+|u|)^{b_{1}}(1+|v|)^{b_{2}}} .
$$

An index $\vec{b}=\left(b_{1}, b_{2}\right)$ is said to be admissible if

$$
b_{1}>\frac{n}{p \wedge q}, \quad b_{2}>\frac{m}{p \wedge q} .
$$

We point out again that the flag multiparameter structure is involved in the definition of Peetre's maximal functions. The maximal function characterizations of Besov spaces and Triebel-Lizorkin spaces are as follows.

Theorem 1.5. Let $\alpha, \beta \in \mathbb{R}$ and $p, q \in(1, \infty)$. If $\vec{b}$ is admissible, then for $f \in \mathcal{S}_{\mathfrak{F}}{ }^{\prime}$, one has

(i) $\|f\|_{\mathcal{B}_{p, q,(1)}^{\alpha, \beta}}:=\left\|S_{0} f\right\|_{p}+\left\|\left\{2^{\alpha j} 2^{\beta k}\left\|\psi_{\vec{b}, j, k}^{*}(f)\right\|_{p}\right\}_{j, k}\right\|_{\ell^{q}} \sim\|f\|_{\mathcal{B}_{p, q}^{\alpha, \beta,}}$

(ii) $\|f\|_{\mathcal{F}_{p, q,(1)}^{\alpha, \beta}}:=\left\|S_{0} f\right\|_{p}+\|\|\left\{2^{\alpha j} 2^{\beta k} \psi_{\vec{b}, j, k}^{*}(f)\right\}_{j, k}\left\|_{\ell^{q}}\right\|_{L^{p}} \sim\|f\|_{\mathcal{F}_{p, q}^{\alpha, \beta}}$

Here and in what follows, one uses the following notation:

$$
\left\|\left\{a_{j, k}\right\}_{j, k}\right\|_{\ell^{q}}:=\left\{\sum_{j=1}^{\infty} \sum_{k=1}^{\infty}\left|a_{j, k}\right|^{q}\right\}^{1 / q} .
$$

In order to state our result for flag singular integrals, we need to recall some definitions given in [2]. Following closely from [2], we begin with the definitions of a class of 
distributions on an Euclidean space $\mathbb{R}^{d}$. A $k$-normalized bump function on a space $\mathbb{R}^{d}$ is a $C^{k}$ function supported on the unit ball with $C^{k}$ norm bounded by 1 . As pointed out in [2], the definitions given below are independent of the choices of $k$, and thus we will simply refer to "normalized bump function" without specifying $k$.

Definition 1.6. A flag kernel on $\mathbb{R}^{n} \times \mathbb{R}^{m}$ is a distribution $K$ on $\mathbb{R}^{n+m}$ which coincides with a $C^{\infty}$ function away from the coordinate subspaces $(0, y)$, where $(0, y) \in \mathbb{R}^{n} \times \mathbb{R}^{m}$ and satisfies

(1) (Differential inequalities) for any multi-indices $\alpha$ and $\beta$,

$$
\left|\partial_{x}^{\alpha} \partial_{y}^{\beta} K(x, y)\right| \leq C_{\alpha, \beta}|x|^{-n-|\alpha|}(|x|+|y|)^{-m-|\beta|},
$$

for all $(x, y) \in \mathbb{R}^{n} \times \mathbb{R}^{m}$ with $|x| \neq 0$,

(2) (Cancellation condition)

$$
\left|\int_{\mathbb{R}^{m}} \partial_{x}^{\alpha} K(x, y) \phi_{1}(\delta y) d y\right| \leq C_{\alpha}|x|^{-n-|\alpha|}
$$

for all multi-index $\alpha$ and every normalized bump function $\phi_{1}$ on $\mathbb{R}^{m}$ and every $\delta>0$,

$$
\left|\int_{\mathbb{R}^{n}} \partial_{y}^{\beta} K(x, y) \phi_{2}(\delta x) d x\right| \leq C_{\beta}|y|^{-m-|\beta|}
$$

for every multi-index $\beta$ and every normalized bump function $\phi_{2}$ on $\mathbb{R}^{n}$ and every $\delta>0$,

$$
\left|\int_{\mathbb{R}^{n+m}} K(x, y) \phi_{3}\left(\delta_{1} x, \delta_{2} y\right) d x d y\right| \leq C,
$$

for every normalized bump function $\phi_{3}$ on $\mathbb{R}^{+m}$ and every $\delta_{1}>0$ and $\delta_{2}>0$.

The boundedness of flag singular integrals on these inhomogeneous Besov spaces and Triebel-Lizorkin spaces is given by the following theorem, whose proof is quite similar to that in homogeneous case in [8]. We omit the proof here.

Theorem 1.7. Suppose that $T$ is a flag singular integral defined on $\mathbb{R}^{n} \times \mathbb{R}^{m}$ with the flag kernel $K$, then, for $p, q>1$ and $\alpha, \beta \in \mathbb{R}, T$ is bounded on $\mathcal{\mp}_{p, q}^{\alpha, \beta}$ and on $\boldsymbol{B}_{p, q}^{\alpha, \beta}$.

As in the classical inhomogeneous Besov spaces and Triebel-Lizorkin spaces, we will give the difference characterization for $B_{p, q}^{\alpha, \beta}$ and $\mathcal{F}_{p, q}^{\alpha, \beta}$. However, the new feature is that the differences of functions are associated with the "flag." More precisely, for $(u, v) \in \mathbb{R}^{n} \times \mathbb{R}^{m}$ and $w \in \mathbb{R}^{m}$, we define the first flag difference (associated the flag $\{(0,0)\} \subset\{(0, y)\}$ ) in $\mathbb{R}^{n} \times \mathbb{R}^{m}$ by

$$
\begin{aligned}
\Delta_{u, v ; w}^{\mathfrak{F}} f(x, y) & =\left[\Delta_{u, v} \circ \Delta_{w}^{(2)}\right] f(x, y) \\
& =f(x+u, y+v+w)-f(x+u, y+v)-f(x, y+w)+f(x, y),
\end{aligned}
$$


where $\Delta_{u, v}$ is the difference operator on $\mathbb{R}^{m+n}$, and $\Delta_{w}^{(2)}$ is the difference operator on $\mathbb{R}^{m}$. For $k \in \mathbb{Z}_{+}$and $k \geq 2$, the $k$ th flag difference operator $\left(\Delta_{u, v ; w}^{\mathfrak{F}}\right)^{k}$ can be defined inductively by

$$
\left(\Delta_{u, v ; w}^{\mathfrak{F}}\right)^{k}=\Delta_{u, v ; w}^{\mathfrak{F}} \circ\left(\Delta_{u, v ; w}^{\mathfrak{F}}\right)^{k-1}
$$

Theorem 1.8. If $\alpha, \beta>0,1<p<\infty, 1<q<\infty$, and $M \geq[\alpha \vee \beta]+1$, where [.] denotes the greatest integer function, one defines

$$
\begin{aligned}
& \|f\|_{\mathcal{B}_{p, q,(2)}^{\alpha, \beta}}:=\|f\|_{p}+\left\{\int_{\mathbb{R}^{n+m} \times \mathbb{R}^{m}}\left(\frac{\left\|\left(\Delta_{u, v ; w}^{\mathfrak{F}}\right)^{M} f\right\|_{p}}{|(u, v)|^{\alpha}|w|^{\beta}}\right)^{q} \frac{d u d v d w}{|(u, v)|^{n+m}|w|^{m}}\right\}^{1 / q}, \\
& \|f\|_{\mathcal{F}_{p, q,(2)}^{\alpha, \beta}}:=\|f\|_{p}+\left\|\left\{\int_{\mathbb{R}^{n+m} \times \mathbb{R}^{m}}\left(\frac{\left|\left(\Delta_{u, v ; w}^{\mathfrak{F}}\right)^{M} f\right|}{|(u, v)|^{\alpha}|w|^{\beta}}\right)^{q} \frac{d u d v d w}{|(u, v)|^{n+m}|w|^{m}}\right\}^{1 / q}\right\|_{p},
\end{aligned}
$$

then $\|f\|_{\mathcal{B}_{p, q,(2)}^{\alpha, \beta}} \sim\|f\|_{\mathcal{B}_{p, q}^{\alpha, \beta},}\|f\|_{\mathcal{F}_{p, q,(2)}^{\alpha, \beta}} \sim\|f\|_{\mathcal{F}_{p, q}^{\alpha, \beta}}$

As mentioned before, by slightly modifying the proof, we can prove difference characterizations and Peetre's maximal function characterizations of homogeneous Besov and Triebel-Lizorkin spaces, introduced in [8]. We leave the details to the interested reader.

The following of the paper is organized as follows. In Section 2, we give some lemmas. The proof of Theorems 1.3 and 1.5 is presented in Section 3. Section 4 is devoted to the proof of Theorem 1.8 .

\section{Some Lemmas}

In this section, we present some lemmas, which will be used in the proofs of the theorems.

\subsection{Inhomogeneous Calderón's Reproducing Formula in $\mathcal{S}_{\mathfrak{F}}^{\prime}$}

Lemma 2.1. The inhomogeneous Calderón's reproducing formula holds

$$
f=\varphi * \varphi * f+\sum_{j=1}^{\infty} \sum_{k=1}^{\infty} \psi_{j, k} * \psi_{j, k} * f
$$

where the series converges in $L^{p}\left(\mathbb{R}^{n+m}\right)(1<p<\infty), \mathcal{S}_{\mathfrak{F}}\left(\mathbb{R}^{n} \times \mathbb{R}^{m}\right)$ and $\mathcal{S}_{\mathfrak{F}}^{\prime}\left(\mathbb{R}^{n} \times \mathbb{R}^{m}\right)$.

We point out that in [8] the homogeneous Calderón's reproducing formula was provided. Note that the convergence of these two kind of producing formulas are different. See [8] for homogeneous case. 
Proof. For any $f \in \mathcal{S}_{\mathfrak{F}}\left(\mathbb{R}^{n} \times \mathbb{R}^{m}\right)$, then by definition, there exists $f^{\#} \in \mathcal{S}\left(\mathbb{R}^{n+m} \times \mathbb{R}^{m}\right)$ such that $f(x, y)=\int_{\mathbb{R}^{m}} f^{\#}(x, y-z, z) d z$. We need to show that for all $N \in \mathbb{Z}_{+}$,

$$
\varphi^{\#} * \varphi^{\#} * f^{\#}+\sum_{\substack{j, k \in \mathbb{Z}_{+}: \\ j \leq N \text { or } k \leq N}} \psi_{j, k}^{\#} * \psi_{j, k}^{\#} * f^{\#},
$$

tends to $f$ in the topology of $\mathcal{S}\left(\mathbb{R}^{n+2 m}\right)$ as $N \rightarrow+\infty$. We only consider the case when $k<N$ in the summation in (2.2) since the other case can be dealt with in the same way. Denote, in this case, the expression (2.2) by $f_{N}^{\#}$. By Fourier inversion,

$$
f^{\#}(x, y)-f_{N}^{\#}(x, y)=\left(\widehat{f}^{\#}-\widehat{f}_{N}^{\#}\right)^{\vee}(x, y), \quad \text { for every }(x, y) \in \mathbb{R}^{n+m} \times \mathbb{R}^{m} .
$$

Let $h_{N}(\xi, \eta, x, y)=e^{i(x \cdot \xi+y \cdot \eta)} \widehat{f}^{\#}(\xi, \eta) \sum_{k=N+1}^{\infty}\left|\widehat{\psi^{(2)}}\left(2^{-k} \eta\right)\right|^{2}$ so that

$$
f^{\#}(x, y)-f_{N}^{\#}(x, y)=c_{n} \int_{\mathbb{R}^{n+m} \times \mathbb{R}^{m}} h_{N}(\xi, \eta, x, y) d \eta d \xi .
$$

Since $\left|h_{N}(\xi, \eta, x, y)\right| \leq\left|\widehat{f}^{\#}(\xi, \eta)\right| \in L^{1}\left(\mathbb{R}^{n+m} \times \mathbb{R}^{m}\right)$, Lebesgue's dominated convergence theorem yields

$$
\lim _{N \rightarrow+\infty} f_{N}^{\#}(x, y)=f^{\#}(x, y), \quad \text { for every }(x, y) \in \mathbb{R}^{n+m} \times \mathbb{R}^{m}
$$

On the other hand, using the cancellation conditions of $\psi_{j}^{(1)} * \psi_{j}^{(1)}, \psi_{k}^{(2)} * \psi_{k}^{(2)}$, and the smoothness of $f^{\#}$, we can get

$$
\left|\psi_{j, k}^{\#} * \psi_{j, k}^{\#} * f^{\#}(x, y)\right| \lesssim 2^{-j} 2^{-k}(1+|x|+|y|)^{-L}, \quad \forall j, k, L \in \mathbb{Z}_{+} .
$$

Now, (2.5) together with the estimate (2.6) implies that, for $f \in \mathcal{S}_{\mathfrak{F}}\left(\mathbb{R}^{n} \times \mathbb{R}^{m}\right)$,

$$
\left|f^{\#}(x)-f_{N}^{\#}(x, y)\right|=\sum_{j \in \mathbb{Z}_{+} k \geq N+1}\left|\psi_{j, k}^{\#} * \psi_{j, k}^{\#} * f^{\#}(x, y)\right| \lesssim 2^{-N}(1+|x|+|y|)^{-L} .
$$

Applying this to $\partial^{u} f^{\#}$ (here $u$ denotes any multi-index in $\mathbb{N}^{n+2 m}$ ) and noting that $\partial^{u} f_{N}^{\#}=$ $\left(\partial^{u} f^{\#}\right)_{N}$, we obtain

$$
\lim _{N \rightarrow+\infty} \sup _{(x, y) \in \mathbb{R}^{n} \times \mathbb{R}^{m}}(1+|x|+|y|)^{L}\left|\partial^{u}\left(f^{\#}-f_{N}^{\#}\right)(x, y)\right|=0 .
$$

This proves the convergence of series in (2.1) in $\mathcal{S}_{\mathfrak{F}}\left(\mathbb{R}^{n} \times \mathbb{R}^{m}\right)$. The convergence in $\mathcal{S}_{\mathfrak{F}}^{\prime}\left(\mathbb{R}^{n} \times \mathbb{R}^{m}\right)$ follows by a standard duality argument. The convergence in $L^{p}\left(\mathbb{R}^{n+m}\right)$ can be proved similar to the product case, see [10, Theorem 1.1]. 


\subsection{Almost Orthogonality Estimates}

The following lemma is the almost orthogonality estimates, which will be frequently used. See [7] for a proof.

Lemma 2.2. Let $x \in \mathbb{R}^{n}, y \in \mathbb{R}^{m}$. Given any positive integers $L$ and $M$, there exists a constant $C=C(L, M)>0$ such that

$$
\left|\psi_{j, k} * \varphi_{j^{\prime}, k^{\prime}}(x, y)\right| \leq C 2^{-\left|j-j^{\prime}\right| L} 2^{-\left|k-k^{\prime}\right| L} \frac{2^{\left(j \wedge j^{\prime}\right) M}}{\left(2^{j \wedge j^{\prime}}+|x|\right)^{n+M}} \frac{2^{\left(j \wedge j^{\prime} \wedge k \wedge k^{\prime}\right) M}}{\left(2^{j \wedge j^{\prime} \wedge k \wedge k^{\prime}}+|y|\right)^{m+M}},
$$

where $\psi, \varphi$ are defined as in Section 1.

\subsection{Maximal Function Estimates}

The maximal function estimates are given as follows.

Lemma 2.3. For $j, k, j^{\prime}, k^{\prime} \in \mathbb{Z}_{+}$, and for any $L>0$ and $\vec{b}=\left(b_{1}, b_{2}\right) \in \mathbb{R}_{+} \times \mathbb{R}_{+}$, there exists a constant $C=C(L, \vec{b})$ depending only on $L$ and $\vec{b}$, but independent on $j, k, j^{\prime}, k^{\prime}$, such that

$$
\left|\left(\psi_{j, k} * \psi_{j^{\prime}, k^{\prime}} * \psi_{j^{\prime}, k^{\prime}} * f\right)(x, y)\right| \leq C 2^{-L\left|j-j^{\prime}\right|} 2^{-L\left|k-k^{\prime}\right|} \psi_{\vec{b}, j^{\prime}, k^{\prime}}^{*}(f)(x, y) .
$$

Proof. By the almost orthogonality estimate in Lemma 2.2, for any $L>0$ and $M>b_{1} \vee b_{2}$, we have the pointwise estimate

$$
\begin{aligned}
& \left|\left(\psi_{j, k} * \psi_{j^{\prime}, k^{\prime}} * \psi_{j^{\prime}, k^{\prime}} * f\right)(x, y)\right| \\
& \lesssim 2^{-\left|j-j^{\prime}\right| L^{\prime}} 2^{-\left|k-k^{\prime}\right| L^{\prime}} \\
& \times \int_{\mathbb{R}^{n} \times \mathbb{R}^{m}} \frac{2^{\left(j \wedge j^{\prime}\right) n} 2^{\left(j \wedge k \wedge j^{\prime} \wedge k^{\prime}\right) m}\left|\psi_{j^{\prime}, k^{\prime}} * f(x-u, y-v)\right|}{\left(1+2^{j \wedge j^{\prime}}|u|\right)^{M+n}\left(1+2^{j \wedge k \wedge j^{\prime} \wedge k^{\prime}}|v|\right)^{M+m}} d u d v \\
& \leq 2^{-\left|j-j^{\prime}\right| L^{\prime}} 2^{-\left|k-k^{\prime}\right| L^{\prime}} \psi_{\vec{b}, j^{\prime}, k^{\prime}}^{*}(f)(x) \\
& \times \int_{\mathbb{R}^{n} \times \mathbb{R}^{m}} \frac{2^{\left(j \wedge j^{\prime}\right) n} 2^{\left(j \wedge k \wedge j^{\prime} \wedge k^{\prime}\right) m}\left(1+2^{j^{\prime}}|u|\right)^{b_{1}}\left(1+2^{j^{\prime} \wedge k^{\prime}}|v|\right)^{b_{2}}}{\left(1+2^{j \wedge j^{\prime}}|u|\right)^{M+n}\left(1+2^{j \wedge k \wedge j^{\prime} \wedge k^{\prime}}|v|\right)^{M+m}} d u d v \\
& \lesssim 2^{-\left|j-j^{\prime}\right|\left(L^{\prime}-b_{1}\right)} 2^{-\left|k-k^{\prime}\right|\left(L^{\prime}-b_{1}-b_{2}\right)} \psi_{\vec{b}, j^{\prime}, k^{\prime}}^{*}(f)(x, y) \\
& \times \int_{\mathbb{R}^{n} \times \mathbb{R}^{m}} \frac{2^{\left(j \wedge j^{\prime}\right) n} 2^{\left(j \wedge k \wedge j^{\prime} \wedge k^{\prime}\right) m}}{\left(1+2^{j \wedge j^{\prime}}|u|\right)^{M+n-b_{1}}\left(1+2^{j \wedge k \wedge j^{\prime} \wedge k^{\prime}}|v|\right)^{M+m-b_{2}}} d u d v \\
& \lesssim 2^{-\left|j-j^{\prime}\right|(L)} 2^{-\left|k-k^{\prime}\right|(L)} \psi_{\vec{b}, j^{\prime}, k^{\prime}}^{*}(f)(x, y) \text {, }
\end{aligned}
$$

where $L=L^{\prime}-b_{1}-b_{2}$. This proves (2.10).

Remark 2.4. Since the almost orthogonality estimates hold with $\psi_{0,0}$ or $\theta_{0,0}$ is replaced by $\varphi$, repeating the same argument as (2.11), we see that the estimate (2.10) is still valid if $\psi_{0,0}$ or $\theta_{0,0}$ is replaced by $\varphi$. 
Journal of Function Spaces and Applications

Denote by $\mathcal{M}_{s}$ the strong maximal operator defined by

$$
\mathcal{M}_{S}(f)(x)=\sup _{R \ni x} \int_{R}|f(y)| d y
$$

where the supremum is taken over all open rectangles $R$ in $\mathbb{R}^{n} \times \mathbb{R}^{m}$ that contain the point $x$.

Lemma 2.5. Let $0<c_{1}, c_{2}<\infty$, and $0<r<\infty$, then for all $j, k \in \mathbb{Z}$ and for all $C^{1}$ functions $u$ on $\mathbb{R}^{n} \times \mathbb{R}^{m}$ whose Fourier transform is supported in the rectangle $\left\{\xi:\left|\xi^{\prime}\right| \leq c_{1} 2^{j},\left|\xi_{n}\right| \leq c_{2} 2^{j \wedge k}\right\}$, one has the estimate

$$
\sup _{y \in \mathbb{R}^{n}} \frac{|u(x-u, y-v)|}{\left(1+2^{j}|u|\right)^{n / r}\left(1+2^{j \wedge k}|v|\right)^{m / r}} \leq C \mathcal{M}_{S}\left(|u|^{r}\right)(x, y)^{1 / r} .
$$

In particular, if $\vec{b}=\left(b_{1}, b_{2}\right)$ with $b_{1} \geq n / r$ and $b_{2} \geq m / r$, then for all $f \in L^{p}\left(\mathbb{R}^{n} \times \mathbb{R}^{m}\right)(1<p<\infty)$,

$$
\psi_{\vec{b}, j, k}^{*}(f)(x, y) \lesssim \mathcal{M}_{s}\left(\left|\psi_{j, k} * f\right|^{r}\right)(x, y)^{1 / r}
$$

Lemma 2.5 can be proved as in the classical one-parameter case. We refer the reader to [11].

\subsection{An Embedding Result}

The following lemma is an embedding result.

Lemma 2.6. For $\alpha, \beta>0$ and $p, q \in(1, \infty)$, one has the following continuous embedding:

$$
B_{p, q}^{\alpha, \beta} \hookrightarrow L^{p}, \quad \mathcal{F}_{p, q}^{\alpha, \beta} \hookrightarrow L^{p}
$$

Proof. For $f \in \mathcal{S}_{\mathfrak{F}}\left(\mathbb{R}^{n} \times \mathbb{R}^{m}\right)$, by inhomogeneous Calderón's reproducing formula (2.1),

$$
\|f\|_{p} \lesssim\left\|S_{0} f\right\|_{p}+\sum_{j=1}^{\infty} \sum_{k=1}^{\infty}\left\|\psi_{j, k} * f\right\|_{p} \lesssim\left\|S_{0} f\right\|_{p}+\left(\sum_{j=1}^{\infty} \sum_{k=1}^{\infty} 2^{j \alpha q} 2^{k \beta q}\left\|\psi_{j, k} * f\right\|_{p}^{q}\right)^{1 / q} \sim\|f\|_{\mathcal{B}_{p, q}^{\alpha, \beta}}
$$

where we have used Hölder's inequality in the last inequality. This proves Lemma 2.6 for Besov spaces. 
For the Triebel-Lizorkin spaces, by (2.1), the pointwise inequality $\left|\psi_{j, k} * f(x, y)\right| \leq$ $\psi_{\vec{b}, j, k}^{*}(f)(x, y)$, Hölder's inequality, and Fefferman-Stein's vector-valued inequality, we have

$$
\begin{aligned}
\|f\|_{p} & \lesssim\left\|\varphi *\left(S_{0} f\right)\right\|_{p}+\left\|\sum_{j=1}^{\infty} \sum_{k=1}^{\infty}\left|\psi_{j, k} * \psi_{j, k} * f\right|\right\|_{p} \\
& \lesssim\left\|S_{0} f\right\|_{p}+\left\|\left(\sum_{j=1}^{\infty} \sum_{k=1}^{\infty} 2^{j \alpha q} 2^{k \beta q} \psi_{\vec{b}, j, k}^{*}(f)^{q}\right)^{1 / q}\right\|_{p} \\
& \lesssim\left\|S_{0} f\right\|_{p}+\left\|\left(\sum_{j=1}^{\infty} \sum_{k=1}^{\infty} 2^{j \alpha q} 2^{k \beta q}\left|\psi_{j, k} * f\right|^{q}\right)^{1 / q}\right\|_{p} \\
& \sim\|f\|_{\mathcal{F}_{p, q}^{\alpha, \beta} .}
\end{aligned}
$$

This ends the proof of Lemma 2.6.

\section{Proof of Theorems 1.3 and 1.5}

We first prove Theorems 1.3 and 1.5 for Triebel-Lizorkin spaces by showing

$$
\|f\|_{\mathcal{F}_{p, q}^{\alpha, \beta}}^{\psi} \lesssim\|f\|_{\mathcal{F}_{p, q,(1)}^{\alpha, \beta}}^{\psi} \lesssim\|f\|_{\mathcal{F}_{p, q, q}^{\alpha, \beta}}^{\Theta} \lesssim\|f\|_{\mathcal{F}_{p, q}^{\alpha, \beta}}^{\theta}
$$

The first inequality in (3.1) follows from the pointwise inequality

$$
\left|\psi_{j, k} * f(x, y)\right| \leq \psi_{\vec{b}, j, k}^{*}(f)(x, y), \quad \forall j, k \in \mathbb{Z}_{+}
$$

Next, for any admissible $b$, fix $\vec{b}$. Since $\vec{b}$ is admissible, we can choose $r \leq p \wedge q$ such that $b_{1} \geq n / r, b_{2} \geq m / r$, and the thus inequality (2.14) holds. We apply Lemma 2.5 and the $L^{p / r}\left(\ell^{q / r}\right)$ boundedness of $\boldsymbol{M}_{s}$ to deduce

$$
\begin{aligned}
\|f\|_{\mathcal{F}_{p, q,(1)}^{\alpha, \beta}}^{\theta} & \lesssim\left\|\left\{2^{j \alpha} 2^{k \beta} \theta_{\vec{b}, j, k}^{*}(f)\right\}_{j, k}\right\|_{L^{p}\left(\ell^{q}\right)} \\
& \lesssim\left\|\left\{2^{j \alpha} 2^{k \beta} M_{s}\left(\left|\theta_{j, k} * f\right|^{r}\right)^{1 / r}\right\}_{j, k}\right\|_{L^{p}\left(\ell^{q}\right)} \lesssim\|f\|_{\mathcal{F}_{p, q}^{\alpha, \beta},}^{\theta},
\end{aligned}
$$

which gives the third inequality in (3.1). 
Thus, to finish the proof of (3.1), it remains to verify the second inequality. For $j, k \in \mathbb{Z}_{+}$, $\phi * \theta_{j, k}$ is nonzero only when $j=1$ and $k=1$. Thus, applying Calderón's identity (2.1), Minkowski's inequality, Remark 2.4, and Lemma 2.5, we deduce that

$$
\begin{aligned}
\|\varphi * f\|_{p} & \lesssim\|\varphi * \phi\|_{1} \cdot\|\phi * f\|_{p}+\left\|\theta_{\vec{b}, 1,1}^{*}(f)\right\|_{p} \\
& \lesssim\|\phi * f\|_{p}+\left\|\left\{2^{j \alpha} 2^{k \beta} \theta_{\vec{b}, j, k}^{*}(f)\right\}_{j, k}\right\|_{L^{p}\left(\ell^{q}\right)} \lesssim\|f\|_{\bar{q}_{p, q,(1)}^{\alpha, \beta}}^{\theta} .
\end{aligned}
$$

To finish the proof, it remains to show

$$
\left\|\left\{2^{j \alpha} 2^{k \beta} \psi_{\vec{b}, j, k}^{*}(f)\right\}_{j, k}\right\|_{L^{p}(\ell q)} \lesssim\|f\|_{\Phi_{p, q,(1)}^{\alpha, \beta}}^{\theta} .
$$

By the inhomogeneous Calderón's identity (2.1), we have

$$
\left|\psi_{j, k} * f(x, y)\right| \leq\left(\left|\psi_{j, k} * \phi\right| *|\phi * f|\right)(x, y)+\sum_{j^{\prime}, k^{\prime} \in \mathbb{Z}_{+}}\left(\left|\psi_{j, k} * \theta_{j^{\prime}, k^{\prime}}\right| *\left|\theta_{j^{\prime}, k^{\prime}} * f\right|\right)(x, y) .
$$

It follows that

$$
\begin{aligned}
& \left\|\left\{2^{j \alpha} 2^{k \beta} \psi_{\vec{b}, j, k}^{*}(f)\right\}_{j, k}\right\|_{L^{p}\left(\ell^{q}\right)} \\
& \leq\left\|\left\{2^{j \alpha} 2^{k \beta} \sup _{(u, v) \in \mathbb{R}^{n} \times \mathbb{R}^{m}} \frac{\left(\left|\psi_{j, k} * \phi\right| *|\phi * f|\right)(\cdot-u, \cdot v)}{\left(1+2^{j}|u|\right)^{b_{1}}\left(1+2^{j \wedge k}|v|\right)^{b_{2}}}\right\}_{j, k}\right\|_{L^{p}\left(\ell^{q}\right)} \\
& \quad+\left\|\left\{2^{j \alpha} 2^{k \beta} \sum_{j^{\prime}, k^{\prime}>0} \sup _{(u, v) \in \mathbb{R}^{n} \times \mathbb{R}^{m}} \frac{\left(\left|\psi_{j, k} * \theta_{j^{\prime}, k^{\prime}}\right| *\left|\theta_{j^{\prime}, k^{\prime}} * f\right|\right)(\cdot-u, \cdot v)}{\left(1+2^{j}|u|\right)^{b_{1}}\left(1+2^{j \wedge k}|v|\right)^{b_{2}}}\right\}_{j, k}\right\|_{L^{p}\left(\ell^{q}\right)} \\
& :=I_{1}+I_{2} .
\end{aligned}
$$

We first estimate $I_{1}$. By the support properties of $\psi_{j, k}$ and $\varphi$ and Young's inequality,

$$
I_{1} \lesssim\left\|\psi_{1,1} * \phi\right\|_{1} \cdot\|\phi * f\|_{p} \lesssim\|\phi * f\|_{p}
$$


Next, we give the estimate for $I_{2}$. For any $(u, v) \in \mathbb{R}^{n} \times \mathbb{R}^{m}$,

$$
\begin{aligned}
& \frac{\left(\left|\psi_{j, k} * \theta_{j^{\prime}, k^{\prime}}\right| *\left|\theta_{j^{\prime}, k^{\prime}} * f\right|\right)(x-u, y-v)}{\left(1+2^{j}|u|\right)^{b_{1}}\left(1+2^{j \wedge k}|v|\right)^{b_{2}}} \\
& \lesssim \int_{\mathbb{R}^{n} \times \mathbb{R}^{m}} \frac{\left|\psi_{j, k} * \theta_{j^{\prime}, k^{\prime}}\left(u^{\prime}, v^{\prime}\right)\right|\left|\theta_{j^{\prime}, k^{\prime}} * f\left(x-u-u^{\prime}, y-v-v^{\prime}\right)\right|}{\left(1+2^{j}|u|\right)^{b_{1}}\left(1+2^{j \wedge k}|v|\right)^{b_{2}}} d u^{\prime} d v^{\prime} \\
& \leq \theta_{\vec{b}, j^{\prime}, k^{\prime}}^{*}(f)(x, y) \int_{\mathbb{R}^{n} \times \mathbb{R}^{m}}\left|\psi_{j, k} * \theta_{j^{\prime}, k^{\prime}}\left(u^{\prime}, v^{\prime}\right)\right| \\
& \times \frac{\left(1+2^{j^{\prime}}\left|u+u^{\prime}\right|\right)^{b_{1}}}{\left(1+2^{j}|u|\right)^{b_{1}}} \frac{\left(1+2^{j^{\prime} \wedge k^{\prime}}\left|v+v^{\prime}\right|\right)^{b_{2}}}{\left(1+2^{j \wedge k}|v|\right)^{b_{2}}} d u d v \\
& \lesssim 2^{-L\left|j-j^{\prime}\right|} 2^{-L\left|k-k^{\prime}\right|} \theta_{\vec{b}, j^{\prime}, k^{\prime}}^{*}(f)(x, y) \text {, }
\end{aligned}
$$

where we have used Lemma 2.2 in the last inequality. Applying Minkowski's inequality and Hölder's inequality yields

$$
\begin{aligned}
& \left\|\left\{2^{j \alpha} 2^{k \beta} \sum_{j^{\prime}, k^{\prime}>0} \sup _{(u, v) \in \mathbb{R}^{n} \times \mathbb{R}^{m}} \frac{\left(\left|\psi_{j, k} * \theta_{j^{\prime}, k^{\prime}}\right| *\left|\theta_{j^{\prime}, k^{\prime}} * f\right|\right)(x-u, y-v)}{\left(1+2^{j}|u|\right)^{b_{1}}\left(1+2^{j \wedge k}|v|\right)^{b_{2}}}\right\}_{j, k}\right\|_{\ell^{q}} \\
& \lesssim\left\{\sum_{j^{\prime}, k^{\prime} \in \mathbb{Z}_{+}}\left(\sum_{j, k \in \mathbb{Z}_{+}} 2^{-\left|j-j^{\prime}\right| q(L-\varepsilon-|\alpha|)} 2^{-\left|k-k^{\prime}\right| q(L-\varepsilon-|\beta|)}\right)\right. \\
& \left.\times 2^{j^{\prime} \alpha q} 2^{k^{\prime} \beta q} \theta_{\vec{b}, j^{\prime}, k^{\prime}}^{*}(f)(x, y)^{q}\right\}^{1 / q} \\
& \lesssim\left\|\left\{2^{j^{\prime} \alpha} 2^{k^{\prime} \beta} \theta_{\vec{b}, j^{\prime}, k^{\prime}}^{*}(f)(x, y)\right\}_{j^{\prime}, k^{\prime}}\right\|_{\ell^{q}},
\end{aligned}
$$

where we have chosen $\varepsilon$ as a small positive constant less than $L-(|\alpha| \vee|\beta|)$. Therefore,

$$
I_{2} \lesssim\left\|\left\{2^{j^{\prime} \alpha} 2^{k^{\prime} \beta} \theta_{\vec{b}, j^{\prime}, k^{\prime}}^{*}(f)\right\}_{j^{\prime}, k^{\prime}}\right\|_{L^{p}\left(\ell^{q}\right)} .
$$

Combining the estimates (3.8) and (3.11), we obtain (3.5). This finishes the proof of (3.1), and hence, Theorems 1.3 and 1.5 for Triebel-Lizorkin spaces follow.

The proofs of Theorems 1.3 and 1.5 for Besov spaces are similar. By (3.2) and the maximal function estimate (2.14), $\left\|\psi_{j, k} * f\right\|_{p} \sim\left\|\psi_{\vec{b}, j, k}^{*}(f)\right\|_{p}$. The conclusion of Theorem 1.5 for Besov spaces follows. By Calderón's reproducing formula (2.1), Young's inequality, 
Journal of Function Spaces and Applications

the almost orthogonality estimate in Lemma 2.2, Hölder's inequality, and Minkowski's inequality, we have

$$
\begin{aligned}
\sum_{j=1}^{\infty} \sum_{k=1}^{\infty} 2^{j \alpha q} 2^{k \beta q}\left\|\psi_{j, k} * f\right\|_{p}^{q} \\
\leq 2^{\alpha q} 2^{\beta q}\left\|\psi_{1,1} * \phi\right\|_{1}^{q}\|\phi * f\|_{p}^{q} \\
\quad+\sum_{j=1}^{\infty} \sum_{k=1}^{\infty} 2^{j \alpha q} 2^{k \beta q}\left(\sum_{j^{\prime}=1}^{\infty} \sum_{k^{\prime}=1}^{\infty}\left\|\psi_{j, k} * \theta_{j^{\prime}, k^{\prime}}\right\|_{1}\left\|\theta_{j^{\prime}, k^{\prime}} * f\right\|_{p}\right)^{q} \\
\lesssim\|\phi * f\|_{p}^{q}+\sum_{j=1}^{\infty} \sum_{k=1}^{\infty} 2^{j \alpha q} 2^{k \beta q}\left(\sum_{j^{\prime}=1}^{\infty} \sum_{k^{\prime}=1}^{\infty} 2^{-(L-\varepsilon) q\left(\left|j-j^{\prime}\right|+\left|k-k^{\prime}\right|\right)}\left\|\theta_{j^{\prime} k^{\prime}} * f\right\|_{p}^{q}\right) \\
\lesssim\|\phi * f\|_{p}^{q}+\sum_{j^{\prime}=1}^{\infty} \sum_{k^{\prime}=1}^{\infty} 2^{j^{\prime} \alpha q} 2^{k^{\prime} \beta q}\left\|\theta_{j^{\prime}, k^{\prime}} * f\right\|_{p^{\prime}}^{q}
\end{aligned}
$$

as desired. This ends the proof of Theorem 1.3 for Besov case. Hence, the proofs of Theorems 1.3 and 1.5 are complete.

\section{Proof of Theorem 1.8}

\subsection{Proof of Theorem 1.8 for Besov Space.}

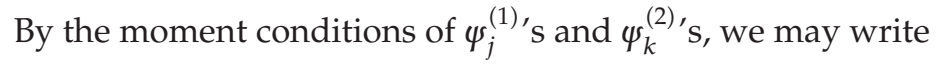

$$
\psi_{j, k} * f(x, y)=\iint_{\mathbb{R}^{n+m} \times \mathbb{R}^{m}} \psi_{j}^{(1)}(u, v) \psi_{k}^{(2)}(w)\left[\left(\Delta_{-u,-v ;-w}^{\mathfrak{F}}\right)^{M} f(x, y)\right] d u d v d w
$$

By Minkowski's inequality and Hölder's inequality and noting that

$$
\left\|\left(\Delta_{-u,-v ;-w}^{\mathfrak{F}}\right)^{M} f\right\|_{p}=\left\|\left(\Delta_{u, v ; w}^{\mathfrak{F}}\right)^{M} f\right\|_{p^{\prime}}
$$

we have

$$
\begin{aligned}
\left\|\psi_{j, k} * f\right\|_{p}^{q} & =\left(\iint_{\mathbb{R}^{n+m} \times \mathbb{R}^{m}}\left|\psi_{j}^{(1)}(u, v)\left\|\psi_{k}^{(2)}(w) \mid\right\|\left(\Delta_{u, v ; w}^{\mathfrak{F}}\right)^{M} f \|_{p} d u d v d w\right)^{q}\right. \\
& \lesssim \iint_{\mathbb{R}^{n+m} \times \mathbb{R}^{m}}\left|\psi_{j}^{(1)}(u, v)\right|\left|\psi_{k}^{(2)}(w)\right|\left\|\left(\Delta_{u, v ; w}^{\mathfrak{F}}\right)^{M} f\right\|_{p}^{q} d u d v d w .
\end{aligned}
$$


Hence,

$$
\begin{aligned}
& \sum_{j=1}^{\infty} \sum_{k=1}^{\infty} 2^{j \alpha q} 2^{k \beta q}\left\|\psi_{j, k} * f\right\|_{p}^{q} \\
& \quad \lesssim \iint_{\mathbb{R}^{n+m} \times \mathbb{R}^{m}}\left(\sum_{j=1}^{\infty} 2^{j \alpha q}\left|\psi_{j}^{(1)}(u, v)\right|\right)\left(\sum_{k=1}^{\infty} 2^{k \beta q}\left|\psi_{k}^{(2)}(w)\right|\right)\left\|\left(\Delta_{u, v ; w}^{\mathfrak{F}}\right)^{M} f\right\|_{p}^{q} d u \mathrm{~d} v d w \\
& \quad \lesssim\|f\|_{\mathbb{B}_{p, q,(2)}^{\alpha, \beta}}^{q,}
\end{aligned}
$$

where the last inequality follows from

$$
\begin{gathered}
\sum_{k=1}^{\infty} 2^{k \beta q}\left|\psi_{k}^{(2)}(w)\right| \lesssim \sum_{k=1}^{\infty} 2^{k \beta q} \frac{2^{-k L}}{\left(2^{-k}+|w|\right)^{m+L}} \lesssim \frac{1}{|w|^{m+\beta q}}, \\
\sum_{j=1}^{\infty} 2^{j \alpha q}\left|\psi_{j}^{(1)}(u, v)\right| \lesssim \frac{1}{|(u, v)|^{n+m+\alpha q}} .
\end{gathered}
$$

This inequality together with the trivial inequality $\left\|S_{0} f\right\|_{p} \lesssim\|f\|_{p}$ yields $\|f\|_{\mathcal{B}_{p, q}^{\alpha, \beta}} \lesssim\|f\|_{\mathcal{B}_{p, q,(2)}^{\alpha, \beta}}$.

To prove the converse, by Lemma 2.6, it suffices to show that

$$
\iint_{\mathbb{R}^{n+m} \times \mathbb{R}^{m}}\left(\frac{\left\|\left(\Delta_{u, v ; w}^{\mathfrak{F}}\right)^{M} f\right\|_{p}}{|(u, v)|^{\alpha}|w|^{\beta}}\right)^{q} \frac{d u d v d w}{|(u, v)|^{n+m}|w|^{m}} \lesssim\|f\|_{\mathcal{B}_{p, q}^{\alpha, \beta}}^{q}
$$

By the Calderón's identity (2.1), we write

$$
\left(\Delta_{u, v ; w}^{\mathfrak{F}}\right)^{M} f=\left(\left(\Delta_{u, v ; w}^{\mathfrak{F}}\right)^{M} \varphi\right) *\left(S_{0} f\right)+\sum_{j=1}^{\infty} \sum_{k=1}^{\infty}\left(\left(\Delta_{u, v ; w}^{\mathfrak{F}}\right)^{M} \psi_{j, k}\right) * \psi_{j, k} * f,
$$

where the series on the right hand side converges in $L^{p}\left(\mathbb{R}^{n+m}\right)$. Thus, by Minkowski's inequality and Young's inequality, we conclude that

$$
\left\|\left(\Delta_{u, v ; w}^{\mathfrak{F}}\right)^{M} f\right\|_{p} \leq\left\|\left(\Delta_{u, v ; w}^{\mathfrak{F}}\right)^{M} \varphi\right\|_{1}\left\|S_{0} f\right\|_{p}+\sum_{j=1}^{\infty} \sum_{k=1}^{\infty}\left\|\left(\Delta_{u, v ; w}^{\mathfrak{F}}\right)^{M} \psi_{j, k}\right\|_{1}\left\|\psi_{j, k} * f\right\|_{p}
$$

It follows that the left hand side in (4.6) is dominated, up to a constant, by the sum of

$$
\begin{gathered}
I I I_{1} \equiv \sum_{j^{\prime} \in \mathbb{Z}} \sum_{k^{\prime} \in \mathbb{Z}} 2^{j^{\prime} \alpha q} 2^{k^{\prime} \beta q}\left\|\left(\Delta_{u, v ; w}^{\mathfrak{F}}\right)^{M} \varphi\right\|_{1}^{q}\left\|S_{0} f\right\|_{p^{\prime}}^{q} \\
I I I_{2} \equiv \sum_{j^{\prime} \in \mathbb{Z}} \sum_{k^{\prime} \in \mathbb{Z}} 2^{j^{\prime} \alpha q} 2^{k^{\prime} \beta q}\left(\sum_{j=1}^{\infty} \sum_{k=1}^{\infty}\left\|\left(\Delta_{u, v ; w}^{\mathfrak{F}}\right)^{M} \psi_{j, k}\right\|_{1}\left\|\psi_{j, k} * f\right\|_{p}\right)^{q} .
\end{gathered}
$$


Journal of Function Spaces and Applications

For $I I I_{1}$, we have

$$
\begin{aligned}
I I I_{1} \lesssim\left\{\begin{array}{l}
\sum_{\substack{j^{\prime}>0 \\
k^{\prime}>0}} 2^{-j^{\prime} q(M-\alpha)} 2^{-k^{\prime} q(M-\beta)}+\sum_{\substack{j^{\prime}>0 \\
k^{\prime} \leq 0}} 2^{-j^{\prime} q(M-\alpha)} 2^{k^{\prime} q \beta} \\
+\sum_{\substack{j^{\prime} \leq 0 \\
k^{\prime}>0}} 2^{j^{\prime} q \alpha} 2^{-k^{\prime} q(M-\beta)}+\sum_{\substack{j^{\prime} \leq 0 \\
k^{\prime} \leq 0}} 2^{j^{\prime} q \alpha} 2^{k^{\prime} q \alpha}
\end{array}\right\}\left\|S_{0} f\right\|_{p}^{q} \lesssim\left\|S_{0} f\right\|_{p^{\prime}}^{q}
\end{aligned}
$$

where we have used the estimate

$$
\left\|\left(\Delta_{u, v ; w}^{\mathfrak{F}}\right)^{M} \varphi\right\|_{1} \lesssim 2^{M \cdot \min \left\{0,-j^{\prime},-k^{\prime},-j^{\prime}-k^{\prime}\right\}}
$$

To estimate $I I I_{2}$, by the estimate

$$
\left\|\left(\Delta_{u, v ; w}^{\mathfrak{F}}\right)^{M} \psi_{j, k}\right\|_{1} \lesssim 2^{M \cdot \min \left\{0, j-j^{\prime}, k-k^{\prime}, j-j^{\prime}+k-k^{\prime}\right\}},
$$

and Hölder's inequality, we see that $I I I_{2}$ is majorized by

$$
\begin{aligned}
& \sum_{j^{\prime}, k^{\prime} \in \mathbb{Z}}\left(\sum_{\substack{0<j \leq j^{\prime} \\
0<k \leq k^{\prime}}} 2^{q[M-\varepsilon-(\alpha \vee \beta)]\left(j-j^{\prime}+k-k^{\prime}\right)}+\sum_{\substack{0<j \leq j^{\prime} \\
k>\left(k^{\prime} \vee 0\right)}} 2^{q\left[(M-\alpha-\varepsilon)\left(j-j^{\prime}\right)+\left(k^{\prime}-k\right)(\beta-\varepsilon)\right]}\right. \\
& \left.\quad+\sum_{\substack{j>\left(j^{\prime} \vee 0\right) \\
0<k \leq k^{\prime}}} 2^{q(M-\beta-\varepsilon)\left(k-k^{\prime}\right)} 2^{q\left(j^{\prime}-j\right)(\alpha-\varepsilon)}+\sum_{\substack{j>\left(j^{\prime} \vee 00\right) \\
k>\left(k^{\prime} \vee 0\right)}} 2^{q\left(j^{\prime}-j\right)(\alpha-\varepsilon)} 2^{q\left(k^{\prime}-k\right)(\beta-\varepsilon)}\right) \\
& \quad \times 2^{j \alpha q} 2^{k \beta q}\left\|\psi_{j, k} * f\right\|_{p}^{q} \\
& \lesssim \sum_{j>0} \sum_{k>0} 2^{j \alpha q} 2^{k \beta q}\left\|\psi_{j, k} * f\right\|_{p^{\prime}}^{q}
\end{aligned}
$$

proving (4.6), where $\varepsilon$ is a positive number such that $0<\varepsilon<(\alpha \wedge \beta)<(\alpha \vee \beta)+\varepsilon<M$. This concludes the proof of Theorem 1.8 for Besov spaces. 


\subsection{Proof of Theorem 1.8 for Triebel-Lizorkin Space.}

Using the moment conditions on $\psi_{j, k}$, we have

$$
\begin{aligned}
& \sum_{j, k \in \mathbb{Z}_{+}} 2^{j \alpha q} 2^{k \beta q}\left|\psi_{j, k} * f(x, y)\right|^{q} \\
& \quad \leq \sum_{j, k \in \mathbb{Z}_{+}} 2^{j \alpha q} 2^{k \beta q} \iint_{\mathbb{R}^{n+m} \times \mathbb{R}^{m}}\left|\tilde{\psi}_{j}^{(1)}(u, v)\right|\left|\tilde{\psi}_{k}^{(2)}(w)\right|\left|\left(\Delta_{u, v ; w}^{\mathfrak{F}}\right)^{M} f(x, y)\right|^{q} d u d v d w \\
& \quad \leq \iint_{\mathbb{R}^{n+m} \times \mathbb{R}^{m}}\left(\sum_{j=1}^{\infty} 2^{j \alpha q}\left|\psi_{j}^{(1)}(u, v)\right|\right)\left(\sum_{k=1}^{\infty} 2^{k \beta q}\left|\psi_{k}^{(2)}(w)\right|\right)\left|\left(\Delta_{u, v ; w}^{\mathfrak{F}}\right)^{M} f(x, y)\right|^{q} d u d v d w \\
& \quad \lesssim \iint_{\mathbb{R}^{n+m} \times \mathbb{R}^{m}}\left(\frac{\left|\left(\Delta_{u, v ; w}^{\mathfrak{F}}\right)^{M} f(x, y)\right|}{|(u, v)|^{\alpha}|w|^{\beta}}\right)^{q} \frac{d u d v d w}{|(u, v)|^{n+m}|w|^{m}},
\end{aligned}
$$

where $\tilde{g}(x):=g(-x)$. Therefore,

$$
\left\|\left(\sum_{j=1}^{\infty} \sum_{k=1}^{\infty} 2^{j \alpha q} 2^{k \beta q}\left|\psi_{j, k} * f\right|^{q}\right)^{1 / q}\right\|_{p} \lesssim\|f\|_{\mathcal{F}_{p, q,(2)}^{\alpha, \beta},},
$$

which, together with the obvious inequality $\left\|S_{0} f\right\|_{p} \lesssim\|f\|_{p}$, yields $\|f\|_{\mathcal{F}_{p, q}^{\alpha, \beta}} \lesssim\|f\|_{\mathcal{F}_{p, q,(2)}^{\alpha, \beta}}$.

To show the converse, write

$$
\left(\Delta_{u, v ; w}^{\mathfrak{F}}\right)^{M} f=\left(\left(\Delta_{u, v ; w}^{\mathfrak{F}}\right)^{M} \varphi\right) *\left(\mathrm{~S}_{0} f\right)+\sum_{j=1}^{\infty} \sum_{k=1}^{\infty}\left(\left(\Delta_{u, v ; w}^{\mathfrak{F}}\right)^{M} \psi_{j, k}\right) * \psi_{j, k} * f,
$$

where the series converges in $\mathcal{S}_{\mathfrak{F}}^{\prime}\left(\mathbb{R}^{n} \times \mathbb{R}^{m}\right)$. It follows that

$$
\begin{aligned}
& \left\|\left\{\iint_{\mathbb{R}^{n+m} \times \mathbb{R}^{m}}\left(\frac{\left|\left(\Delta_{u, v ; w}^{\mathfrak{F}}\right)^{M} f\right|}{|(u, v)|^{\alpha}|w|^{\beta}}\right)^{q} \frac{d u d v d w}{|(u, v)|^{n+m}|w|^{m}}\right\}^{1 / q}\right\|_{p} \\
& \leq\left\|\left\{\sum_{j^{\prime} \in \mathbb{Z}} \sum_{k^{\prime} \in \mathbb{Z}} 2^{j^{\prime} \alpha q} 2^{k^{\prime} \beta q}\left|\left(\left(\Delta_{u, v ; w}^{\mathfrak{F}}\right)^{M} \varphi\right) * S_{0} f\right|^{q}\right\}^{1 / q}\right\|_{p} \\
& \quad+\|\left\{\sum_{j^{\prime} \in \mathbb{Z}} \sum_{k^{\prime} \in \mathbb{Z}} 2^{j^{\prime} \alpha q} 2^{k^{\prime} \beta q}\left(\sum_{j=1}^{\infty} \sum_{k=1}^{\infty}\left|\left(\left(\Delta_{u, v ; w}^{\mathfrak{F}}\right)^{M} \psi_{j, k}\right) * \psi_{j, k} * f\right|\right)^{q 1 / q} \|\right. \\
& :=I V_{1}+I V_{2},
\end{aligned}
$$

where $|(u, v)| \sim 2^{-j^{\prime}}$ and $|w| \sim 2^{-k^{\prime}}$. 
Journal of Function Spaces and Applications

For $I V_{2}$, by Lemma 2.2,

$$
\left|\left(\Delta_{u, v ; w}^{\mathfrak{F}}\right)^{M} \psi_{j, k}(u, v)\right| \lesssim 2^{M \cdot \min \left\{0, j-j^{\prime}, k-k^{\prime}, j-j^{\prime}+k-k^{\prime}\right\}} \frac{2^{j n} 2^{(j \wedge k) m}}{\left(1+2^{j}|u|+2^{j \wedge k}|v|\right)^{L_{2}}} .
$$

Consequently,

$$
\left|\left(\Delta_{u, v ; w}^{\mathfrak{F}}\right)^{M} \psi_{j, k} * \psi_{j, k} * f(x, y)\right| \lesssim 2^{M \cdot \min \left\{0, j-j^{\prime}, k-k^{\prime}, j-j^{\prime}+k-k^{\prime}\right\}} \psi_{\vec{b}, j, k}^{*}(f)(x, y) .
$$

Therefore,

$$
\begin{gathered}
\sum_{j^{\prime} \in \mathbb{Z} k^{\prime} \in \mathbb{Z}} 2^{j^{\prime} \alpha q} 2^{k^{\prime} \beta q}\left(\sum_{j=1}^{\infty} \sum_{k=1}^{\infty}\left|\left(\left(\Delta_{u, v ; w}^{\mathfrak{F}}\right)^{M} \psi_{j, k}\right) * \psi_{j, k} * f(x, y)\right|\right)^{q} \\
\lesssim \sum_{j, k \in \mathbb{Z}_{+}}\left(\sum_{\substack{j^{\prime}>j \\
k^{\prime}>k}} 2^{q[M-\varepsilon-(\alpha \vee \beta)]\left(j-j^{\prime}+k-k^{\prime}\right)}+\sum_{\substack{j^{\prime}>j \\
k^{\prime}<k}} 2^{q(M-\alpha-\varepsilon)\left(j-j^{\prime}\right)} 2^{q\left(k^{\prime}-k\right)(\beta-\varepsilon)}\right. \\
\left.+\sum_{\substack{j^{\prime}<j \\
k^{\prime}>k}} 2^{q(M-\beta-\varepsilon)\left(k-k^{\prime}\right)} 2^{q\left(j^{\prime}-j\right)(\alpha-\varepsilon)}+\sum_{\substack{j^{\prime}<j \\
k^{\prime}<k}} 2^{q\left(j^{\prime}-j\right)(\alpha-\varepsilon)} 2^{q\left(k^{\prime}-k\right)(\beta-\varepsilon)}\right) \\
\quad \times 2^{j \alpha q} 2^{k \beta q} \psi_{\vec{b}, j, k}^{*}(f)(x, y)^{q} \\
\lesssim \sum_{j, k \in \mathbb{Z}_{+}} 2^{j \alpha q} 2^{k \beta q} \psi_{\vec{b}, j, k}^{*}(f)(x, y)^{q},
\end{gathered}
$$

which together with the maximal function characterization of $\mathcal{F}_{p, q}^{\alpha, \beta}$ implies

$$
I V_{2} \lesssim\left\|\left\{\sum_{j, k \in \mathbb{Z}_{+}} 2^{j \alpha q} 2^{k \beta q} \Psi_{\vec{b}, j, k}^{*}(f)^{q}\right\}^{1 / q}\right\|_{p} \lesssim\|f\|_{\mathcal{F}_{p, q}^{\alpha, \beta}}
$$

As for $I V_{1}$, similar estimate to (4.19) yields

$$
\left|\left(\Delta_{u, v ; w}^{\mathfrak{F}}\right)^{M} \varphi * S_{0} f(x, y)\right| \lesssim 2^{-M \cdot \max \left\{0, j^{\prime}, k^{\prime}, j^{\prime}+k^{\prime}\right\}} \psi_{\vec{b}, 0,0}^{*}(f)(x, y)
$$


Therefore,

$$
\begin{aligned}
I V_{1} \lesssim\left\{\begin{array}{c}
\sum_{\substack{j^{\prime}>0 \\
k^{\prime}>0}} 2^{-j^{\prime} q(M-\alpha)} 2^{-k^{\prime} q(M-\beta)}+\sum_{\substack{j^{\prime}>0 \\
k^{\prime} \leq 0}} 2^{-j^{\prime} q(M-\alpha)} 2^{k^{\prime} q \beta} \\
\\
\quad+\sum_{\substack{j^{\prime} \leq 0 \\
k^{\prime}>0}} 2^{j^{\prime} q \alpha} 2^{-k^{\prime} q(M-\beta)}+\sum_{\substack{j^{\prime} \leq 0 \\
k^{\prime} \leq 0}} 2^{j^{\prime} q \alpha} 2^{k^{\prime} q \alpha}
\end{array}\right\}\left\|\psi_{\vec{b}, 0,0}^{*}(f)\right\|_{p} \lesssim\left\|S_{0} f\right\|_{p} .
\end{aligned}
$$

This estimate together with (4.21) and Lemma 2.6 yields $\|f\|_{\mathcal{F}_{p, q,(2)}^{\alpha, \beta}} \lesssim\|f\|_{\mathcal{F}_{p, q}^{\alpha, \beta}}$. This ends the proof of Theorem 1.8 .

\section{Acknowledgments}

The research was supported by NNSF of China (Grant nos. 11101423, 11171345) and the Fundamental Research Funds for the Central Universities of China (Grant no. 2009QS12). The authors would like to express their deep gratitude to the referee for his/her valuable comments and suggestions.

\section{References}

[1] D. Müller, F. Ricci, and E. M. Stein, "Marcinkiewicz multipliers and multi-parameter structure on Heisenberg (-type) groups. I," Inventiones Mathematicae, vol. 119, no. 2, pp. 199-233, 1995.

[2] A. Nagel, F. Ricci, and E. M. Stein, "Singular integrals with flag kernels and analysis on quadratic CR manifolds," Journal of Functional Analysis, vol. 181, no. 1, pp. 29-118, 2001.

[3] A. Nagel, F. Ricci, E. M. Stein, and S. Wainger, "Singular integrals with flag Kernels on homogeneous groups: I," Functional Analysis. In press. http:/ / arxiv.org/abs/1108.0177.

[4] A. Nagel, F. Ricci, E. M. Stein, and S. Wainger, "Singular integrals with flag Kernels on homogeneous groups: II," to appear, 2011.

[5] P. Głowacki, "Composition and $L^{2}$-boundedness of flag kernels," Colloquium Mathematicum, vol. 118, no. 2, pp. 581-585, 2010.

[6] P. Głowacki, " $L^{p}$-boundedness of flag kernels on homogeneous groups," Functional Analysis. In press. http:/ / arxiv.org/pdf/1006.2532.pdf.

[7] Y. Han and G. Lu, "Discrete Littlewood-Paley-Stein Theory and multi-parameter Hardy spaces associated with flag singular integrals," Classical Analysis and ODEs. In press. http:/ /arxiv:0801.1701.

[8] Y. Ding, G. Z. Lu, and B. L. Ma, "Multi-parameter Triebel-Lizorkin and Besov spaces associated with flag singular integrals," Acta Mathematica Sinica (English Series), vol. 26, no. 4, pp. 603-620, 2010.

[9] D. Yang, "Besov and Triebel-Lizorkin spaces related to singular integrals with flag kernels," Revista Matemática Complutense, vol. 22, no. 1, pp. 253-302, 2009.

[10] X. Wu, "Atomic decomposition characterizations of weighted multiparameter Hardyspaces," Frontiers of Mathematics in China. In press.

[11] L. Grafakos, Classical and Modern Fourier Analysis, Pearson/Prentice Hall, Upper Saddle River, NJ, USA, 2004. 


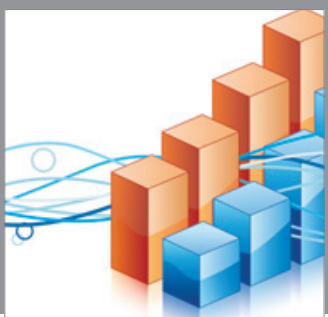

Advances in

Operations Research

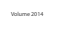

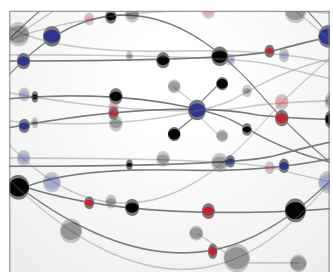

\section{The Scientific} World Journal
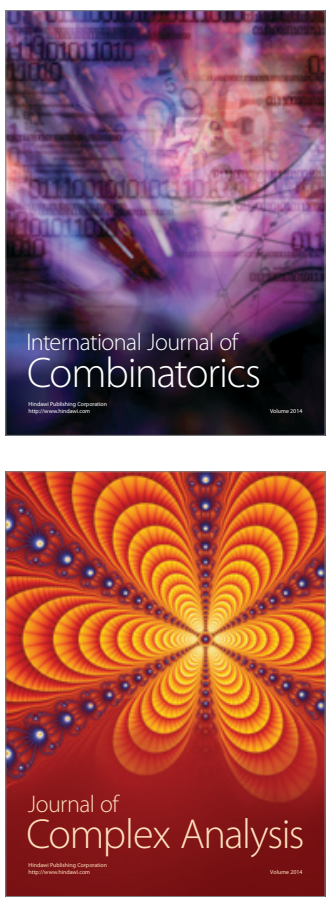

International Journal of

Mathematics and

Mathematical

Sciences
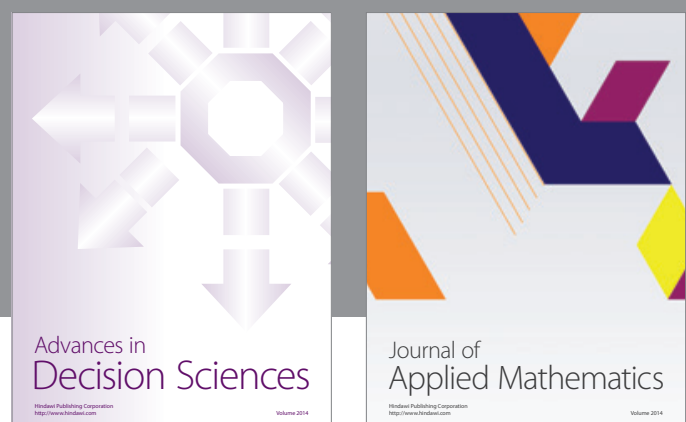

Journal of

Applied Mathematics
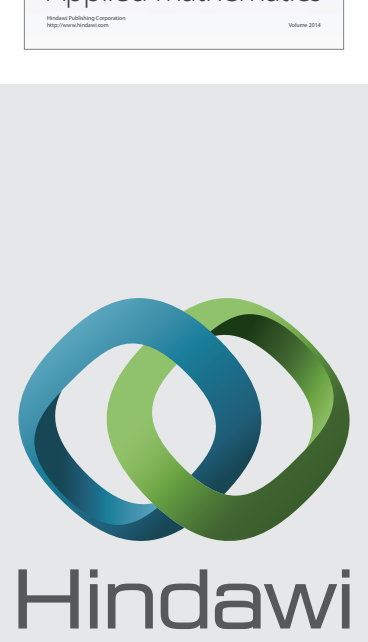

Submit your manuscripts at http://www.hindawi.com
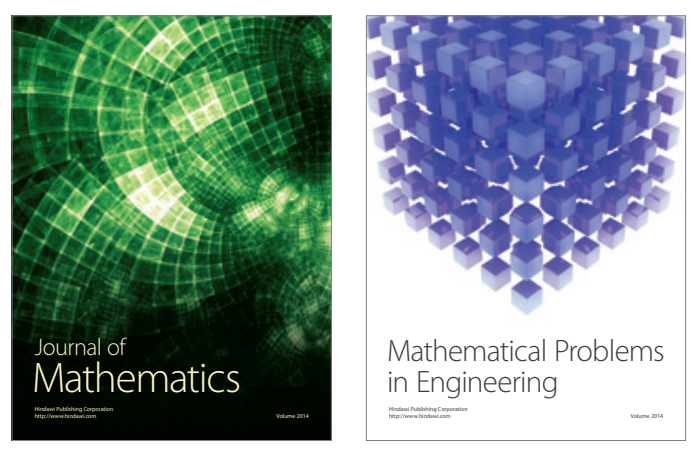

Mathematical Problems in Engineering
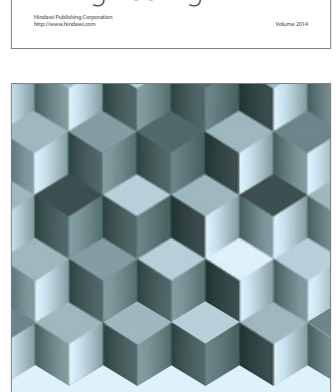

Journal of

Function Spaces
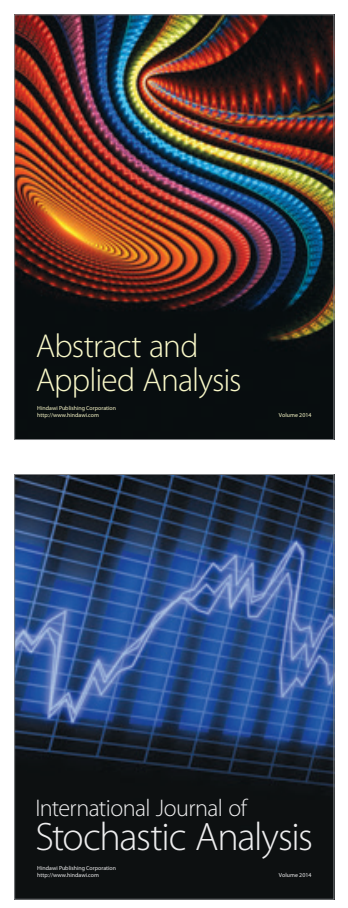

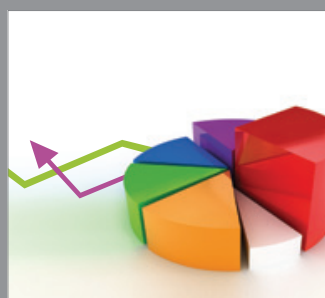

ournal of

Probability and Statistics

Promensencen
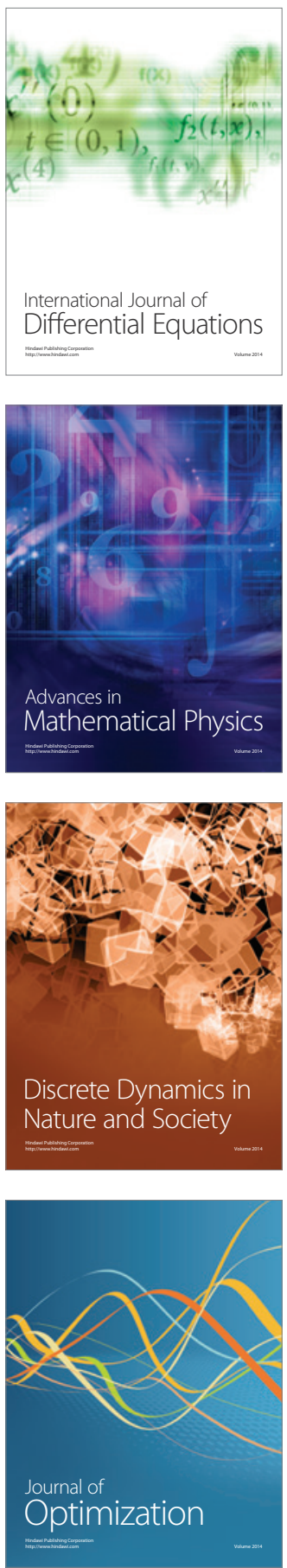